\title{
Erratum: Multicenter integrals over long-range operators using Cartesian Gaussian functions [Phys. Rev. A 37, 2834 (1988)]
}

\author{
Peter Schwerdtfeger (10) and H. Silberbach \\ (Received 28 May 2021; published 15 June 2021)
}

DOI: 10.1103/PhysRevA.103.069901

The group headed by Glover and co-workers [1] came across a rather subtle error in our evaluation of the multicenter integrals over long-range operators using Cartesian Gaussian functions arising from the incorrect use of infinities in the alternating series (39) of our original paper. The correction adds an additional term to (48) and, subsequently, to (37) for the $H(1, a)$ term. To show this, we remember the original limit for the integral in Eq. (34) [subcase 2] and substitute correctly for the integration range $[0, \epsilon] \equiv\left[0, \epsilon_{\eta}\right]=\left[0, \frac{b^{2} \eta}{a_{\mu}^{2}+a_{\mu} \eta}\right]$ for the limit $\eta \rightarrow \infty$. This changes the integration limit of the next equation on top of pg. 2838 accordingly, and we have to solve the slightly modified integral,

$$
\lim _{\eta \rightarrow \infty} \int_{\left[0, \sqrt{\frac{\eta}{a_{\mu}+\eta}}\right]} \frac{x^{2 j}}{\left(1-x^{2}\right)^{p}} e^{-\left(b^{2} / a_{\mu}\right) x^{2}}=\lim _{\eta \rightarrow \infty} h_{\epsilon_{\eta}}\left(j, p, b^{2} / a_{\mu}\right),
$$

with a redefined $\epsilon_{\eta}=\sqrt{\frac{\eta}{a_{\mu}+\eta}}$. With this modification we can carry easily through the equations which follow in the original paper, but we end up with two important modifications. First, we evaluate the limit for the modified Eq. (48) (the original factor $a_{\mu}^{n}$ in this equation is not required as for $p=1$ we have $a_{\mu}^{p-1}=1$ ),

$$
\lim _{\eta \rightarrow \infty} \sum_{\mu=0}^{q}\left(\begin{array}{c}
q \\
\mu
\end{array}\right)(-1)^{\mu} \tanh ^{-1}\left(\epsilon_{\eta}\right)=\frac{1}{2} \lim _{\eta \rightarrow \infty} \sum_{\mu=0}^{q}\left(\begin{array}{c}
q \\
\mu
\end{array}\right)(-1)^{\mu}\left[\ln \left(1+\epsilon_{\eta}\right)-\ln \left(1-\epsilon_{\eta}\right)\right] .
$$

As we have $\lim _{\eta \rightarrow \infty} \epsilon_{\eta}=1$ and, therefore, $\lim _{\eta \rightarrow \infty} \ln \left(1+\epsilon_{\eta}\right)=\ln (2)$, the first term in (2) gives zero for $q \geqslant 1$ according to

$$
\sum_{\mu=0}^{q}\left(\begin{array}{c}
q \\
\mu
\end{array}\right)(-1)^{\mu}=\delta_{q 0}
$$

Hence, it remains to evaluate the limit for the more problematic term for $q \geqslant 1$ (which is always fulfilled for subcase 2 in the original paper),

$$
-\frac{1}{2} \lim _{\eta \rightarrow \infty} \sum_{\mu=0}^{q}\left(\begin{array}{c}
q \\
\mu
\end{array}\right)(-1)^{\mu} \ln \left(1-\epsilon_{\eta}\right)
$$

where we cannot interchange the limit with the sum. Using a Taylor expansion for the argument in the logarithmic term,

$$
\left.\left(1-\epsilon_{\eta}\right)=1-\sqrt{\frac{1}{1+\frac{a_{\mu}}{\eta}}}=\frac{a_{\mu}}{2 \eta}+\text { (higher-order terms }\right)
$$

leads to (neglecting higher-order terms)

$$
-\frac{1}{2} \lim _{\eta \rightarrow \infty} \sum_{\mu=0}^{q}(-1)^{\mu}\left(\begin{array}{c}
q \\
\mu
\end{array}\right)\left[\ln \left(a_{\mu}\right)-\ln (2 \eta)\right]=-\frac{1}{2} \sum_{\mu=0}^{q}(-1)^{\mu}\left(\begin{array}{c}
q \\
\mu
\end{array}\right) \ln \left(a_{\mu}\right)+\frac{1}{2} \lim _{\eta \rightarrow \infty} \sum_{\mu=0}^{q}\left(\begin{array}{c}
q \\
\mu
\end{array}\right)(-1)^{\mu} \ln (2 \eta) .
$$

The second sum is zero for all finite $\eta>0$ and $q \geqslant 1$, and the limit returns a zero result. On the other hand, the result does not change if we substitute $\ln \left(a_{\mu}\right)$ in the sum by $\ln \left(a_{\mu} / b^{2}\right)$. Because of the nonzero sum containing now the $\ln \left(a_{\mu} / b^{2}\right)$ term, we need to modify $H(1, a)$ in Eq. (37) accordingly [we note that the $a \equiv\left(b^{2} / a_{\mu}\right)$ variable in $\left(37^{\prime}\right)$ is $\mu$ dependent]. Hence, $H(1, a)$ as defined in the original paper in (37) becomes

$$
H\left(1, b^{2} / a_{\mu}\right)=e^{-b^{2} / a_{\mu}}\left[2 m\left(\sqrt{b^{2} / a_{\mu}}\right)-\frac{1}{2} \ln \left(a_{\mu} / b^{2}\right)\right],
$$


where $m(x)$ is the Dawson-error hybrid function as defined in our original paper, and we note the additional second term missing in the original equation.

I am very grateful to A. Humeniuk, X. Liu, and W. J. Glover (New York University, Shanghai) for communicating their detailed and very careful analysis of our original 1988 paper.

[1] A. Humeniuk, X. Liu, and W. J. Glover (personal communication). 